\title{
Do bisphosphonates oversuppress bone turnover?
}

Bisphosphonates reduce fracture risk in postmenopausal women with osteoporosis, predominantly via their antiresorptive action on bone, but do they also suppress bone turnover to an extent that bone becomes adynamic and bone quality is compromised? Recent findings of Delmas et al. suggest not.

The effect of three annual infusions of $5 \mathrm{mg}$ zoledronic acid on bone turnover was assessed in a subset of postmenopausal women with osteoporosis enrolled in the multicenter, randomized, HORIZON (Health Outcomes and Reduced Incidence with Zoledronic Acid Once Yearly) trial.

Zoledronic acid treatment substantially reduced bone turnover: 1 year after the last of the three annual infusions, median decreases in serum levels of bone turnover markers were 51\%, 30\% and 56\% for $\beta$-C-terminal telopeptide of type I collagen, bone alkaline phosphatase and procollagen type I N-terminal propeptide
(PINP), respectively. Despite the reductions, most patients' levels of bone turnover markers remained within the normal premenopausal reference range throughout the study.

\section{4 ...it goes against the dogma that bone quality is harmed by 'oversuppression' of bone turnover 77}

The researchers also examined fracture incidence in 1,132 patients whose level of PINP was measured at baseline and 1 year, and discovered something unexpected. "We found that the fracture risk in women with levels of PINP below the reference interval for postmenopausal women was no higher (and in fact, lower) than in women who had levels within the reference interval," says study author Richard Eastell, from the University of Sheffield, UK. "This is an interesting finding as it goes against the dogma that bone quality is harmed by 'oversuppression' of bone turnover." In addition, annual infusions of zoledronic acid did not lead to adynamic bone. The third infusion was followed by a decrease in bone resorption within a few days, but bone turnover bounced back, with a gradual increase over the following year.

Levels of bone turnover markers are useful in clinical practice to monitor the effects of antiresorptive treatments in osteoporosis. As Eastell points out, "these findings indicate that if we find low values then there is no cause for alarm (at least not over the first 3 years of treatment)."

\section{Carol Wilson}

Original article Delmas, P. et al. Effects of yearly zoledronic acid $5 \mathrm{mg}$ on bone turnover markers and relation of PINP with fracture reduction in postmenopausal women with osteoporosis. J. Bone Miner. Res. doi:10.1359/ jbmr.090310 